\title{
Fabry-Pérot interferometry for microplasma diagnostics
}

\author{
H. Hojo \\ Plasma Research Center, University of Tsukuba, Tsukuba 305-8577, Japan
}

\section{A. Mase}

Art, Science and Technology Center for Cooperative Research, Kyushu University, Kasuga 816-8580, Japan

(Received 8 May 2006; presented on 10 May 2006; accepted 9 August 2006; published online 16 October 2006)

A new method for determining the electron density of a thin plasma by means of Fabry-Pérot interferometry is proposed. The interferometer consists of two plasma layers and dielectric material surrounded by two plasma layers. The transmittance of electromagnetic waves across the interferometer is calculated, and Fabry-Pérot resonances are demonstrated. It is shown that the electron density can be determined from the measurement of the Fabry-Pérot resonance frequencies. This method can also be applied to the measurement of conduction electron density in semiconductor films. @ 2006 American Institute of Physics. [DOI: 10.1063/1.2351910]

\section{INTRODUCTION}

A Fabry-Pérot interferometer is often used for spectroscopic measurements of visible light ${ }^{1-3}$ and $\mathrm{x}$ ray. ${ }^{4}$ In the Fabry-Pérot interferometer, the feature of a used étalon is well known to detect appropriate electromagnetic-wave signals. However, we oppositely try to measure the physical quantity of an étalon by detecting the Fabry-Pérot resonance frequency. Namely, we here propose a new method for determining the electron density of a thin plasma by means of Fabry-Pérot interferometry. A modeled Fabry-Pérot interferometer using thin plasmas as the resonator is shown in Fig. 1 . We assume that a uniform plasma is confined by a very thin material that is transparent for electromagnetic waves, but neglect the plasma-confining material, for simplicity. A dielectric material with the dielectric constant $\varepsilon_{a}$ is inserted between two thin-plasma layers. If the wave frequency $\omega$ is larger than the electron plasma frequency $\omega_{\mathrm{pe}}$, wave is a propagating mode in a plasma, and otherwise the wave becomes an evanescent mode. In this article, we show that Fabry-Pérot resonances occur and have very sharp peaks in $\omega$ for wave-evanescent overdense plasmas $\left(\omega<\omega_{\text {pe }}\right)$, and then the Fabry-Pérot interferometer can attain its high resolution. ${ }^{5,6}$ Thus, we can determine the electron density of a thin plasma from the measurement of the resonance frequency because the resonance frequency is dependent on $\omega_{\mathrm{pe}}$.

$$
\left[\frac{d^{2}}{d z^{2}}+k^{2} \varepsilon(\omega, z)\right] E(z)=0,
$$

with

$$
\varepsilon(\omega, z)=\left\{\begin{array}{cc}
1, & z<0 \\
1-\left(\frac{\omega_{\text {pe }}}{\omega}\right)^{2}, & 0 \leqslant z \leqslant h \\
\varepsilon_{a}, & h<z<h+L \\
1-\left(\frac{\omega_{\text {pe }}}{\omega}\right)^{2}, & h+L \leqslant z \leqslant 2 h+L \\
1, & z>2 h+L,
\end{array}\right.
$$

where $k=\omega / c, c$ is the speed of light, $\omega_{\mathrm{pe}}=\left(e^{2} n_{p} / \varepsilon_{0} m\right)^{1 / 2}$, where $n_{p}$ is the electron density, $m$ is the electron mass, $e$ is the electric charge, and $\varepsilon_{0}$ is the permittivity of free space. The solution of Eq. (1) with Eq. (2) is given by, for overdense plasmas $\left(\omega<\omega_{\text {pe }}\right)$,

$$
E=\left\{\begin{array}{cc}
E_{0} e^{i k z}+b e^{-i k z}, & z<0 \\
c_{1} e^{\lambda z}+d_{1} e^{-\lambda z}, & 0 \leqslant z \leqslant h \\
f e^{i k z}+g e^{-i k a^{z}}, & h<z<h+L \\
c_{2} e^{\lambda z}+d_{2} e^{-\lambda z}, & h+L \leqslant z \leqslant 2 h+L \\
a e^{i k z}, & z>2 h+L,
\end{array}\right.
$$

with

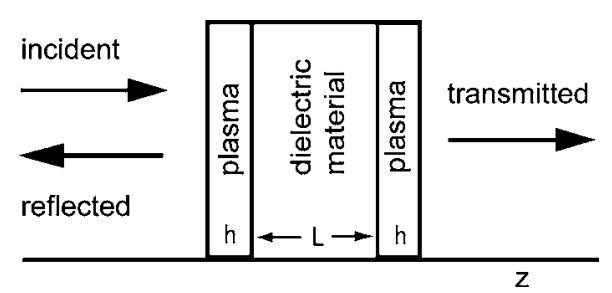

FIG. 1. Schematic of Fabry-Pérot interferometer using thin plasmas.

\section{WAVE TRANSMITTANCE}

We here calculate the transmittance of electromagnetic waves propagating across the modeled Fabry-Pérot interferometer shown in Fig. 1. We consider electromagnetic waves with normal incidence to the Fabry-Pérot interferometer. Thus, our starting point is a one-dimensional Maxwell wave equation given by 


$$
\lambda=k \sqrt{\delta}=k \sqrt{\left(\omega_{\mathrm{pe}} / \omega\right)^{2}-1} \text { and } k_{a}=k \sqrt{\varepsilon_{a}} \text {, }
$$

where the eight coefficients $a, b, c_{1}, c_{2}, d_{1}, d_{2}, f$, and $g$ are determined from the continuity conditions of $E$ and its derivative at $z=0, h, h+L$, and $2 h+L$. After rather lengthy calculations, we can obtain the transmittance $T$ of electromagnetic waves traversing the Fabry-Pérot interferometer as

$$
T=\frac{\left(1 / \varepsilon_{a}\right)[16 \delta /(1+\delta)]^{2}}{S_{1}+S_{2}+S_{3}+S_{4}+S_{5}+S_{6}},
$$

with

$$
\begin{aligned}
& S_{1}=16\left(1+\frac{\delta}{\varepsilon_{a}}\right)^{2} \sin ^{2}\left(k L \sqrt{\varepsilon_{a}}\right) \\
& S_{2}=16 e^{2 k h \sqrt{\delta}}\left(1+\frac{\delta}{\varepsilon_{a}}\right) \frac{1-\delta}{1+\delta} \sin \left(k L \sqrt{\varepsilon_{a}}\right) \\
& \times\left[2 \sqrt{\frac{\delta}{\varepsilon_{a}}} \cos \left(k L \sqrt{\varepsilon_{a}}\right)-\left(1-\frac{\delta}{\varepsilon_{a}}\right) \sin \left(k L \sqrt{\varepsilon_{a}}\right)\right], \\
& S_{3}=-16 e^{-2 k h \sqrt{\delta}}\left(1+\frac{\delta}{\varepsilon_{a}}\right) \frac{1-\delta}{1+\delta} \sin \left(k L \sqrt{\varepsilon_{a}}\right) \\
& \times\left[2 \sqrt{\frac{\delta}{\varepsilon_{a}}} \cos \left(k L \sqrt{\varepsilon_{a}}\right)+\left(1-\frac{\delta}{\varepsilon_{a}}\right) \sin \left(k L \sqrt{\varepsilon_{a}}\right)\right], \\
& S_{4}=2 e^{4 k h \sqrt{\delta}}\left\{\left(1+\frac{\delta}{\varepsilon_{a}}\right)^{2}-4 \sqrt{\frac{\delta}{\varepsilon_{a}}}\left(1-\frac{\delta}{\varepsilon_{a}}\right) \sin \left(2 k L \sqrt{\varepsilon_{a}}\right)\right. \\
& \left.-\left[\left(1-\frac{\delta}{\varepsilon_{a}}\right)^{2}-4 \frac{\delta}{\varepsilon_{a}}\right] \cos \left(2 k L \sqrt{\varepsilon_{a}}\right)\right\} \text {, } \\
& S_{5}=2 e^{-4 k h \sqrt{\delta}}\left\{\left(1+\frac{\delta}{\varepsilon_{a}}\right)^{2}-4 \sqrt{\frac{\delta}{\varepsilon_{a}}}\left(1-\frac{\delta}{\varepsilon_{a}}\right) \sin \left(2 k L \sqrt{\varepsilon_{a}}\right)\right. \\
& \left.+\left[\left(1-\frac{\delta}{\varepsilon_{a}}\right)^{2}-4 \frac{\delta}{\varepsilon_{a}}\right] \cos \left(2 k L \sqrt{\varepsilon_{a}}\right)\right\} \text {, }
\end{aligned}
$$

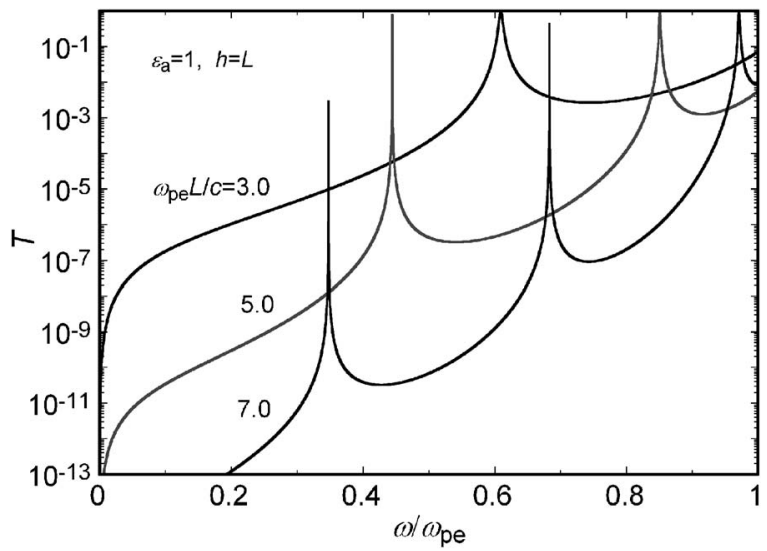

FIG. 2. Transmittance $T$ as a function of $\omega / \omega_{\mathrm{pe}}$ for $\varepsilon_{a}=1, h / L=1$, and $\omega_{\mathrm{pe}} L / c=3,5$, and 7 .

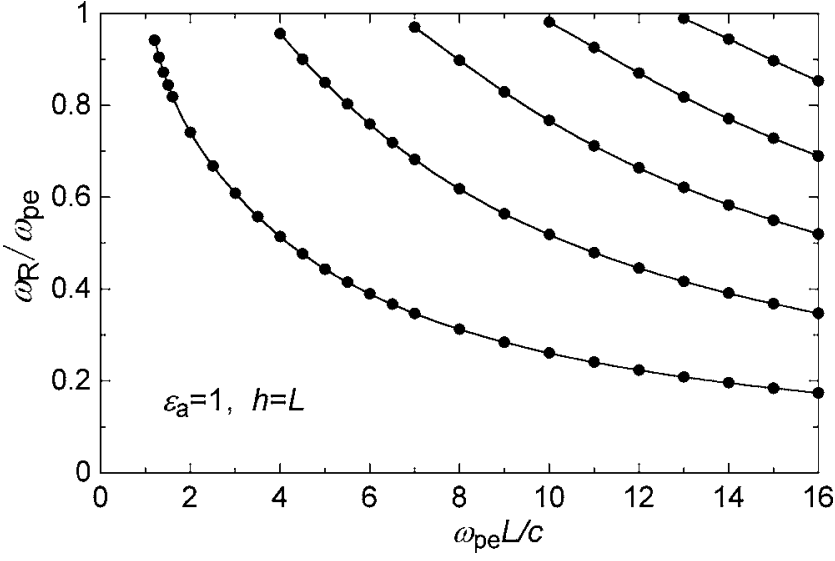

FIG. 3. Resonance frequencies $\omega_{R} / \omega_{\mathrm{pe}}$ as a function of $\omega_{\mathrm{pe}} L / c$ for $\varepsilon_{a}=1$ and $h / L=1$.

$$
\begin{aligned}
S_{6}= & 4 \frac{(1-\delta)^{2}-4 \delta}{(1+\delta)^{2}}\left[\left(1-\frac{\delta}{\varepsilon_{a}}\right)^{2}-4 \frac{\delta}{\varepsilon_{a}}-\left(1+\frac{\delta}{\varepsilon_{a}}\right)^{2}\right. \\
& \left.\times \cos \left(2 k L \sqrt{\varepsilon_{a}}\right)\right] .
\end{aligned}
$$

We see that the transmittance $T$ is a function of four parameters, that is, $k L, k h, \varepsilon_{a}$, and $\delta$; however, it is more convenient for analysis to consider that $T$ is a function of dimensionless parameters: $h / L, \omega / \omega_{\mathrm{pe}}, \varepsilon_{a}$, and $\omega_{\mathrm{pe}} L / c$.

\section{ELECTRON DENSITY MEASUREMENT}

We first show the wave transmittance $T$ as a function of $\omega / \omega_{\mathrm{pe}}$ for $\varepsilon_{a}=1$ (i.e., vacuum), $h / L=1$, and three different values of $\omega_{\mathrm{pe}} L / c(=3,5$, and 7$)$ in Fig. 2. We see that one Fabry-Pérot resonance appears for $\omega_{\mathrm{pe}} L / c=3$, and we have two resonances for $\omega_{\mathrm{pe}} L / c=5$ and three resonances for $\omega_{\mathrm{pe}} L / c=7$. Thus, we find that the number of the resonance increases with the increase of $\omega_{\mathrm{pe}} L / c$, and the resonance peak becomes sharper for the larger value of $\omega_{\mathrm{pe}} L / c$. We show the Fabry-Pérot resonance frequencies as a function of $\omega_{\mathrm{pe}} L / c$ for $\varepsilon_{a}=1$ and $h / L=1$ in Fig. 3. The resonance frequencies normalized by $\omega_{\text {pe }}$ up to the fifth resonance are shown in the figure. Each resonance frequency goes down with the increase of $\omega_{\mathrm{pe}} L / c$.

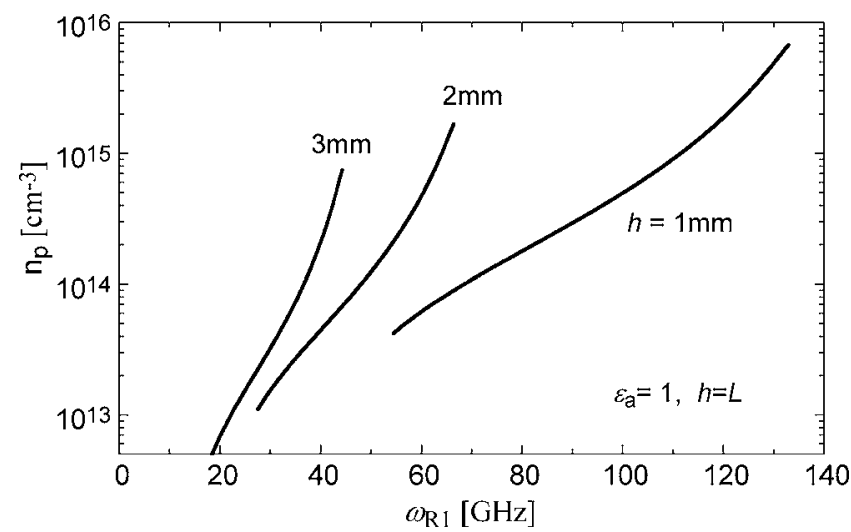

FIG. 4. Electron density $n_{p}$ as a function of the first resonance frequency $\omega_{R 1}$ for $\varepsilon_{a}=1$ and $h=L=1,2$, and $3 \mathrm{~mm}$. 


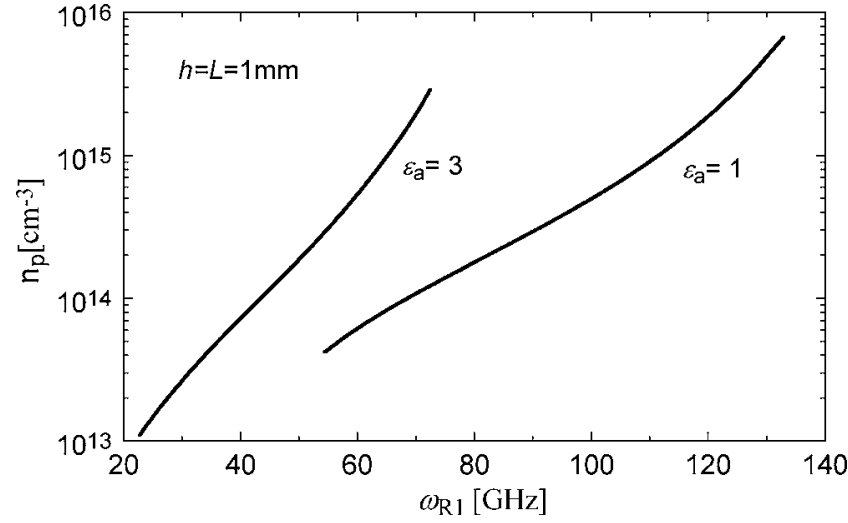

FIG. 5. Plasma density $n_{p}$ as a function of the first resonance frequency $\omega_{R}$ for $\varepsilon_{a}=1$ and 3 , and $h=L=1 \mathrm{~mm}$.

We now discuss a method for determining the electron density of a thin plasma used in the Fabry-Pérot interferometer. We concentrate ourselves to the first resonance frequency, which is primarily detected by upward frequency scanning. As the first resonance frequency $\omega_{R}$ shown in Fig. 3 is a function of $\omega_{\mathrm{pe}}, \varepsilon_{a}, h / L$, and $\omega_{\mathrm{pe}} L / c$, that is,

$$
\omega_{R 1}=\omega_{\mathrm{pe}} f\left(\omega_{\mathrm{pe}} L / c, h / L, \varepsilon_{a}\right),
$$

when $L, h$, and $\varepsilon_{a}$, are known in Eq. (6), we can determine the plasma density $n_{p}$ through $\omega_{\text {pe }}$ by measuring the first resonance frequency $\omega_{R 1}$. We show the relationship between the electron density $n_{p}$ and the first resonance frequency $\omega_{R 1}$ for $\varepsilon_{a}=1$ and different values of $h(=L)$ in Fig. 4. Thus, we see that we can determine the electron density $n_{p}$ from the measurement of the first resonance frequency $\omega_{R 1}$ by the Fabry-Pérot interferometry if the plasma thickness $h$ is known. As shown in Fig. 5, it is also found that the resonance frequency shifts to the lower-frequency side for the fixed $h$ and $n_{p}$ when the dielectric constant $\varepsilon_{a}$ increases. Figure 5 shows that we can control the Fabry-Pérot resonance frequency by changing the value of dielectric constant $\varepsilon_{a}$.

We here emphasize that the present method can also be applied to the measurement of the conduction electron den- sity in semiconductor films. In this case, the model shown in Fig. 1 holds in the form as it is. When we assume the absorption-free Drude model for conduction electrons called as semiconductor plasma in semiconductor films, the dielectric function is given by

$$
\varepsilon(\omega)=\varepsilon_{0}\left[\varepsilon_{\text {core }}-\left(\frac{\omega_{\mathrm{pe}}}{\omega}\right)^{2}\right],
$$

where $\varepsilon_{\text {core }}$ is the background dielectric constant related to valence electrons. The dielectric function of Eq. (7) is similar to that of the present plasma model. Thus, when we replace $\delta$ in Eqs. (4) and (5) by $\left(\omega_{\text {pe }} / \omega\right)^{2}-\varepsilon_{\text {core }}$, the wave transmittance $T$ in Eq. (5) is applicable to the semiconductor plasma. Therefore, we can measure the conduction electron density in thin semiconductor films by the present Fabry-Pérot interferometry.

Finally, we here proposed a new method for measuring the electron density of a thin-plasma layer by the Fabry-Pérot interferometry. The present method can be applied to the density measurement of microplasmas such as plasmadisplay-panel (PDP) plasmas as well as the conduction electron density in semiconductor films. Works on the calculations of electromagnetic-wave transmittance for the more realistic five-layer model such as dielectric-plasmadielectric-plasma-dielectric will be reported elsewhere in the future.

\section{ACKNOWLEDGMENT}

This work was partly supported by the Grant-in-Aid for Scientific Research from the Ministry of Education, Culture, Sports and Technology of Japan.

${ }^{1}$ M. J. Bloemer and M. Scalora, Appl. Phys. Lett. 72, 1676 (1998).

${ }^{2}$ M. Scalora, M. J. Bloemer, A. S. Peeethel, J. P. Dowling, C. M. Bowden, and A. S. Manka, J. Appl. Phys. 83, 2377 (1998).

${ }^{3}$ R. L. Waters and M. E. Aklufi, Appl. Phys. Lett. 81, 3320 (2002).

${ }^{4}$ Yu. V. Shvyd'ko, M. Lerche, H. C. Wille, E. Gerdau, M. Lucht, and H. D. Ruter, Phys. Rev. Lett. 90, 013904 (2003).

${ }^{5}$ H. Hojo and A. Mase, J. Plasma Fusion Res. 80, 358 (2004).

${ }^{6}$ H. Hojo and A. Mase, Extended Abstracts of Optics Japan 2004, Osaka, November 2004, p. 330 